Article

\title{
Investigating the Efficiency Droop of Nitride-Based Blue LEDs with Different Quantum Barrier Growth Rates
}

\author{
C. K. Wang *, Y. Z. Chiou and H. J. Chang \\ Department of Electronic Engineering, Southern Taiwan University of Science and Technology, \\ Tainan City 71005, Taiwan; yzchiou@stust.edu.tw (Y.Z.C.); ma130101@stust.edu.tw (H.J.C.) \\ * Correspondence: ckwang@stust.edu.tw
}

Received: 14 October 2019; Accepted: 13 December 2019; Published: 17 December 2019

check for updates

\begin{abstract}
In this study, GaN-based blue InGaN/GaN light-emitting diodes (LEDs) with different growth rates of the quantum barriers were fabricated and investigated. The LEDs with quantum barriers grown with a higher growth rate exhibit a lower leakage current and less non-radiative recombination centers in the multiple quantum wells (MQWs). Therefore, the LED with a higher barrier growth rate achieves a better light output power by $18.4 \%$ at $120 \mathrm{~mA}$, which is attributed to weaker indium fluctuation effect in the QWs. On the other hand, the localized states created by indium fluctuation lead to a higher local carrier density, and Auger recombination in the QWs. Thus, the efficiency droop ratio of the LEDs with a higher barrier growth rate was only $28.6 \%$, which was superior to that with a lower barrier growth rate $(39.3 \%)$.
\end{abstract}

Keywords: efficiency droop; nitride-based LEDs; quantum barrier; and growth rate

\section{Introduction}

Recently, LEDs have been used for many applications, such as full-color displays, exterior automotive lighting, traffic lights, indicators, and cell phones. LEDs are the most important lighting devices in the future due to their advantages in terms of environmental protection, long lifetime, and vivid color. However, some existing mechanisms of the LEDs can still lead to the efficiency droop effect, such as electron overflow loss [1-3], lacking in hole injection [4,5], Auger recombination [6-8], carrier delocalization [9,10], junction heating [11], and current crowding [12].

In the past several years, many studiees have reported different kinds of electron blocking layer (EBL) of the LEDs in the literature to improve these problems, such as shortperiod superlattice (SPS) EBL with AlGaN/GaN structure, SPS EBL with graded-composition [13-15], and p-AlInGaN or p-AlInN EBL [16], which can inhibit the effect of electron overflow and enhance the ability of hole injection to diminish the efficiency droop effect. Iveland et al. reported the effect of efficiency droop in GaN-based LEDs that originated from the excitation of Auger recombination processes by using electron emission spectroscopy to detect the Auger electrons [6]. Galler et al. reported that when using the recombination rate equation (i.e., $\mathrm{R}=\mathrm{An}+\mathrm{Bn}^{2}+\mathrm{Cn}^{3}, \mathrm{ABC}$ model), the dominant physical mechanism of the efficiency droop was superlinear in the carrier density of electron and can thereby be attributed to Auger recombination [7]. Delaney et al. illustrated that Auger recombination was really an important damage mechanism in the InGaN quantum wells by the calculations of the Auger recombination process [8]. Then, Yang et al. reported that the quantum efficiency and peak shift were dependent on the injection currents and exhibited a significant function of the indium alloy composition in the MQWs. Such a result offered strong evidence that the delocalization was the primary loss mechanism of the efficiency roll-off in InGaN-based LEDs [9]. 
As mentioned previously, the efficiency droop effect limits the use of the LEDs especially, in the case of application of a high current density. Therefore, we need to further improve the effect of efficiency droop of the LEDs. In our study, GaN-based InGaN/GaN MQW blue LEDs with different growth rates of the quantum barriers were fabricated. It is reported by Keller et al. that by using 12 pair MQW structures with $3 \mathrm{~nm}$ thick InGaN well and $4.5 \mathrm{~nm}$ thick GaN barrier, indium at a lower growth rate of GaN barrier can desorb from some areas of the InGaN well, which will cause a lower well indium content and a higher compositional non-uniformity [17]. Therefore, by modulating the growth rates of the quantum barriers, it will be expected that the injection carrier behavior in the MQWs can be improved, thus the quantum efficiency and efficiency droop of the LEDs were also investigated.

\section{Experimental}

The epitaxial layers of the samples used in our study were all grown on c-plane (0001) sapphire substrates by AIXTRON metal-organic chemical vapor deposition (MOCVD) system with Close Coupled Showerhead technology. About the MO source of epitaxial growth, trimethylgallium (TMGa) (Cassen, Hsinchu, Taiwan), trimethylindium (TMIn) (Cassen, Hsinchu, Taiwan), trimethylaluminum (TMAl) (Cassen, Hsinchu, Taiwan), and ammonia (NH3) (Praxair, Hsinchu, Taiwan) were used as gallium, indium, aluminum, and nitrogen sources, respectively. Disilane (Si2H6) (Praxair, Hsinchu, Taiwan) and biscyclopentadienyl magnesium (CP2Mg) (Cassen, Hsinchu, Taiwan) were used as the doping sources of the n- and p-type $\mathrm{GaN}$, respectively. First of all, a $30 \mathrm{~nm}$ thick $\mathrm{GaN}$ buffer layer was grown on top of the sapphire substrate at low temperature of $550{ }^{\circ} \mathrm{C}$. Then, a $3 \mu \mathrm{m}$ thick un-doped $\mathrm{GaN}$ layer and a $2 \mu \mathrm{m}$ thick Si-doped n-GaN layer were grown at $1050^{\circ} \mathrm{C}$. After that, the pre-strain layer with 20 pairs and a lower indium composition InGaN/GaN structure was grown at $900{ }^{\circ} \mathrm{C}$. Then, the carrier recombination centers of InGaN/GaN MQWs were grown. The active region of the MQWs consisted of six pairs of InGaN QW layers with $3 \mathrm{~nm}$ thickness and GaN quantum barrier with $6 \mathrm{~nm}$ thickness, which were grown at 800 and $910{ }^{\circ} \mathrm{C}$, respectively. The growth rates of the GaN quantum barrier layers labeled LED I, LED II, and LED III were 15, 11.25, and $7.5 \AA / \mathrm{min}$, respectively, which were achieved by a change in III/V ratio. Finally, a $30 \mathrm{~nm}$ thick Mg-doped p-AlGaN electron EBL and an $80 \mathrm{~nm}$ thick $\mathrm{Mg}$-doped p-GaN layer were all grown at $1000^{\circ} \mathrm{C}$. In order to activate the p-AlGaN and $\mathrm{p}-\mathrm{GaN}$ layers, the as-grown epitaxial wafers were all further annealed at $650{ }^{\circ} \mathrm{C}$ by the furnace in an N2 ambient atmosphere.

For the process of the LED chip, the chip size was $580 \times 1140 \mu \mathrm{m}^{2}$, which was defined by using standard photolithography. The mesa of the LED chip was performed by dry etching until the $2 \mu \mathrm{m}$ thick n-GaN layer was exposed. Then, the RF sputter system was used to deposit a transparent and conductive indium tin oxide (ITO) layer with $70 \mathrm{~nm}$ thickness onto the $\mathrm{p}-\mathrm{GaN}$ layer, which can serve as the p-type ohmic contact material, current spreading film, and transparent layer. Next, we used the e-beam evaporator to deposit an $\mathrm{Ni} / \mathrm{Au}(30 / 500 \mathrm{~nm})$ metal contact layer as the p-electrode on the ITO layer, and a Ti/Al/Ti/Au (15/450/50/500 nm) metal contact as the n-electrode on the exposed n-GaN layer. After that, the backside of a 2 inch sapphire substrate with epitaxial layers were polished down to about the thickness of $90 \mu \mathrm{m}$. Finally, the LED chips were performed by dicing saw. After the chip process, the TO-39 metal cans without epoxy resin or silicone for LED encapsulation are used to package these fabricated LED chips, which can collect the emission light. The integrating sphere detector (Forter Tech, Tainan, Taiwan) can be used to measure the light output powers (LOPs) of the fabricated LEDs. The current-voltage (I-V) curves of these fabricated LEDs were all measured by a semiconductor parameter analyzer of HP4156C.

\section{Results and Discussion}

The LOPs, external quantum efficiency (EQE), and normalized EQE of the fabricated LEDs on a linear and $\log$ I scale as shown in Figure 1a-c, respectively. As shown in Figure 1a, the LOPs of LED I, LED II, and LED III were 106.6, 100.7, and $90.0 \mathrm{~mW}$ at $120 \mathrm{~mA}$, respectively. In addition, the LOP of LED I at $120 \mathrm{~mA}$ was enhanced by $5.9 \%$ and $18.4 \%$ compared with LED II and LED III, 
respectively. It was evident that LED I exhibited a superior LOP. Otherwise, the LOPs of LED I, LED II, and LED III were 214.2, 192.9, and $172.0 \mathrm{~mW}$ at $300 \mathrm{~mA}$, respectively. As compared with LED II and LED III, the LOP of LED I was improved by approximately $11.0 \%$ and $24.5 \%$ at $300 \mathrm{~mA}$, respectively. The same tendency is also shown in Figure 1b. The differences of LOP and EQE between the fabricated LEDs evidently became significantly larger especially, at higher injection currents. Then, as shown in Figure 1c, normalized EQE can more clearly compare the differences of the efficiency droop. It was found that the EQE decay percentages from maximum EQE to EQE at $300 \mathrm{~mA}$ of LED I, LED II, and LED III were $28.6 \%, 35.1 \%$, and $39.3 \%$, respectively. Such a result once again pointed out that the optical properties of LED I were superior to those of the others whether operating at low or high injection currents. The physical mechanism of this phenomenon will be proposed in the following paragraphs in detail.

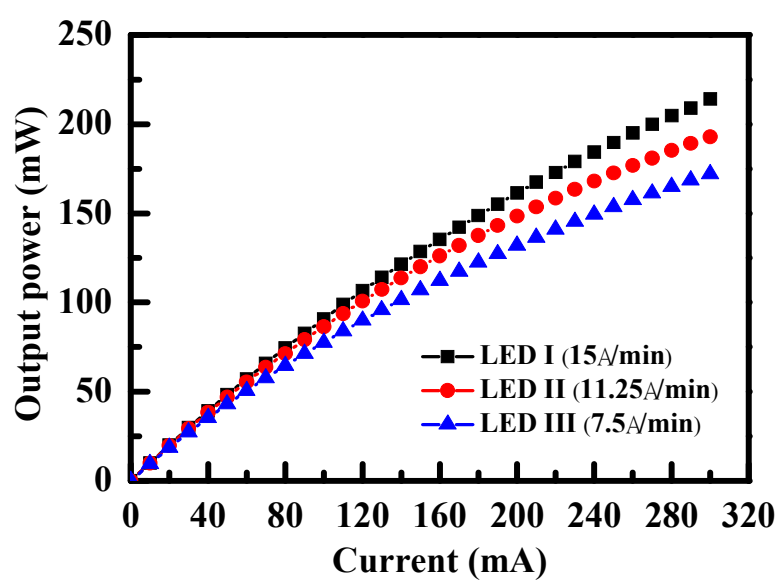

(a)

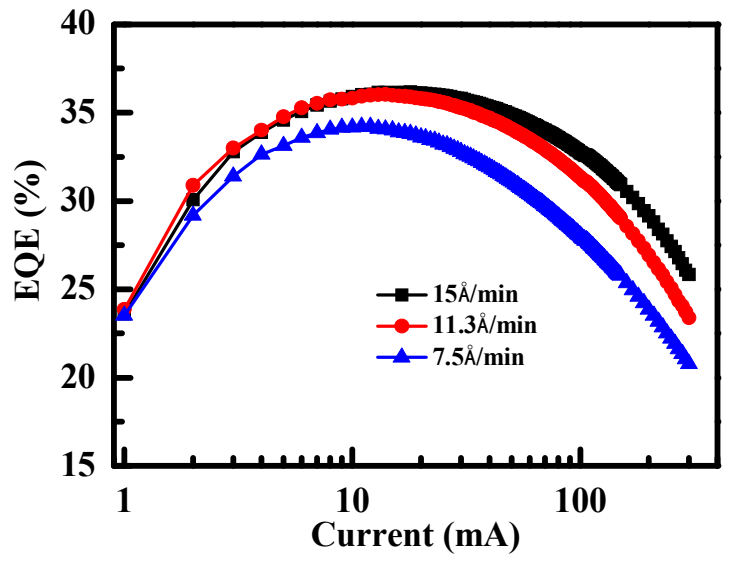

(b)

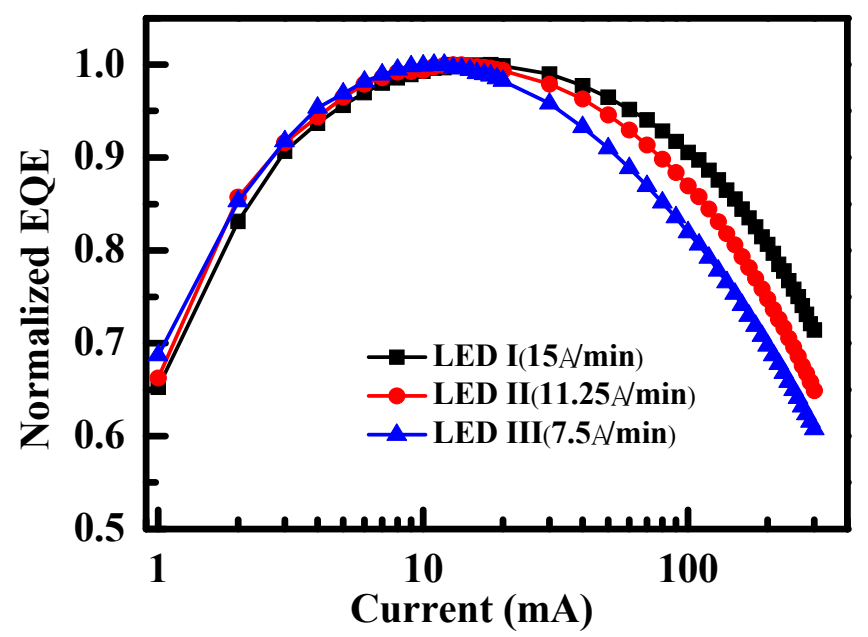

(c)

Figure 1. (a) The light output power, (b) external quantum efficiency (EQE), and (c) normalized EQE of three light-emitting diodes (LEDs) as a function of the injection currents on a linear and $\log$ I scale, respectively.

Figure 2 shows the forward current-voltage characteristics on a semilogarithmic scale. It is obvious that the forward current of LED III under the forward bias voltage of $2.4 \mathrm{~V}$ is larger than that of the other LEDs. According to the equivalent circuit model of the pn junction diode [18], LED IIIwith a smaller parallel resistance-is attributed to a larger leakage current. It is pointed out that the excess current of LEDs generated at a voltage of less than $2.4 \mathrm{~V}$ is attributed to the minority carrier 
recombination by the traps in the depletion region most likely related to the V-defects in the MQWs, which arise from the heterointerfaces between the wells and barriers $[19,20]$. Figure $3 a, b$ shows the (002) and (102) double-crystal X-ray diffraction (DCXRD) spectra of LED I and LED III, respectively. It can be seen clearly that both the main and satellite peaks of (002) XRD spectrum of LED I exhibit larger intensity and narrower full-width at half-maximum (FWHM) than those of LED III in Figure 3a. The same results are also shown in (102) XRD spectrum. The superior X-ray (102) characteristic of LED I implies a lower V-pits density forms along the threading dislocations. Such a result demonstrates that the crystal quality of MQWs of LED I with a higher barrier growth rate is better than that of LED III, which corresponds to the forward I-V characteristics, as mentioned above.

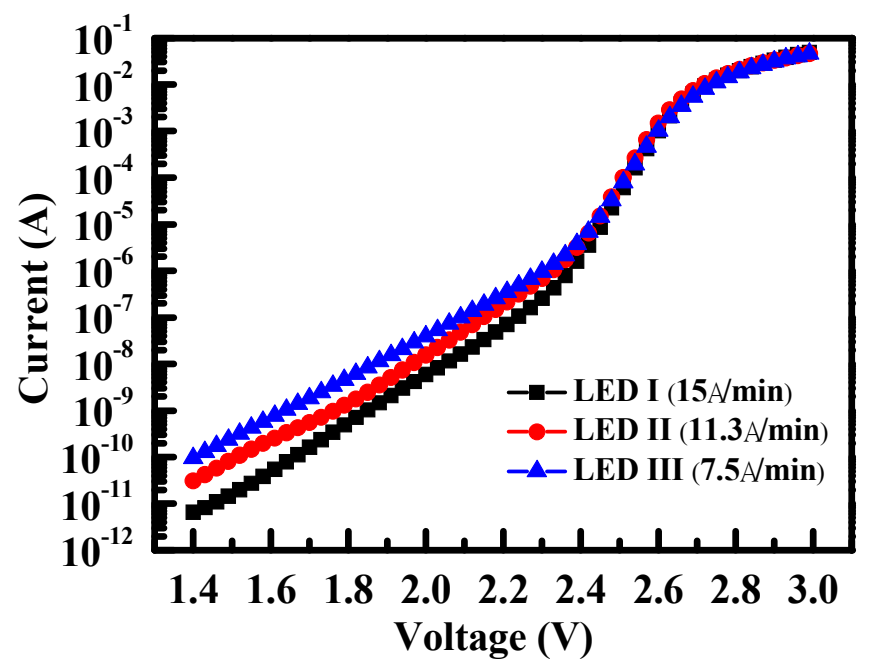

Figure 2. The current characteristics under the forward voltages in a semilogarithmic scale.

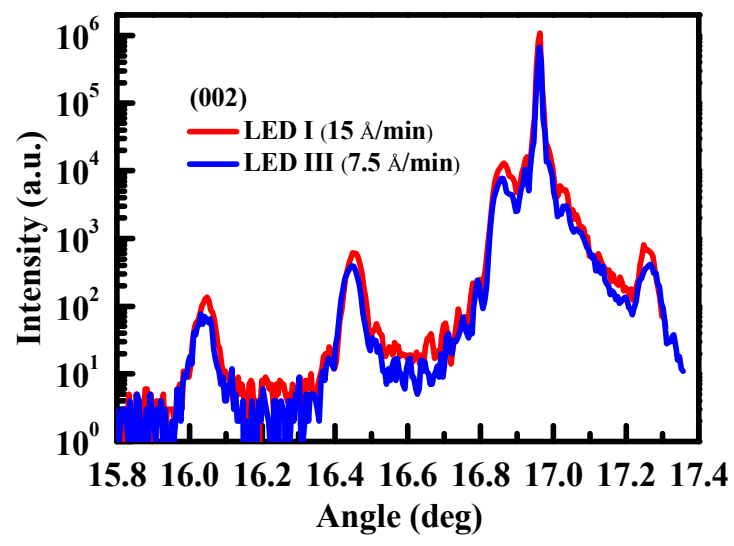

(a)

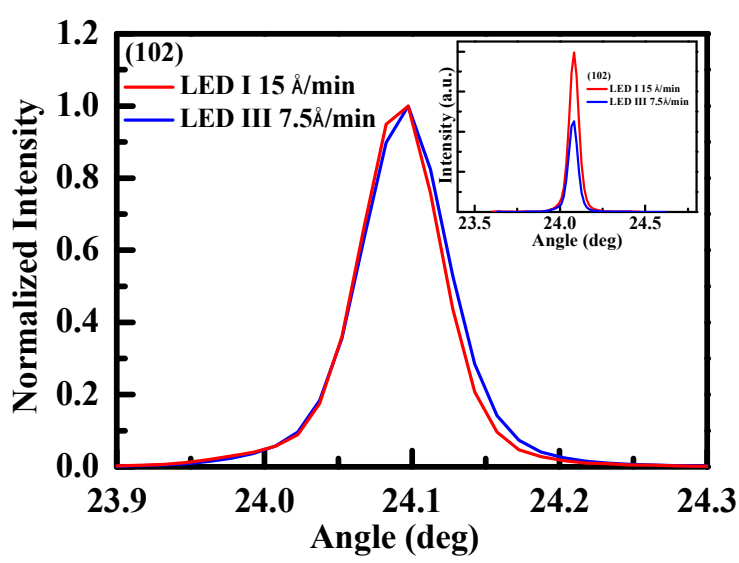

(b)

Figure 3. The (a) (002) and (b) (102) DCXRD spectra for LED I and LED III.

Then, we present an in-depth approach to analyze the non-radiative recombination in the MQW region by the cathodoluminescence (CL) system. The CL spectra of these three LEDs (not shown here) include a weaker GaN near-band-edge (NBE) emission (i.e., $365 \mathrm{~nm}$ ), a stronger $\mathrm{InGaN} / \mathrm{GaN}$ MQWs emission (i.e., $450 \mathrm{~nm}$ ), and a broad yellow band (YL) emission (i.e., $550 \mathrm{~nm}$ ). Figure $4 \mathrm{a}, \mathrm{b}$ shows the CL mapping images of the LED I and III recorded at an MQW emission wavelength of $450 \mathrm{~nm}$, respectively. It is clearly evident that LED I has more light spots than LED III. According to the literature, the V-defects in the MQW can act as non-radiative recombination centers and generate the dark areas in the CL images [21,22]. The results obtained demonstrate that LED III has a greater 
V-defect density and larger leakage current, which also corresponds to the results of Figures 2 and 3. This could be attributed to the fact that the growth temperature of the quantum barriers was $110^{\circ} \mathrm{C}$ higher than that of the quantum wells. In order to suppress the generation of V-pits in the MQWs and enhance the carrier confinement capability of MQWs, the growth temperature of the quantum barrier was higher than that of QW, and growth rate of the quantum barrier was decreased. However, if the growth temperature and rate of the quantum barrier are too high and low, respectively, the indium fluctuation effect $[23,24]$ in the MQWs can be induced, which will result in the defect generation (i.e., non-radiative recombination centers) and crystal quality degradation of the MQWs. On the basis of the above, we choose a compromise, which is the reduction of the growth time (i.e., increase the barrier growth rate appropriately) at $910{ }^{\circ} \mathrm{C}$. LED I increases the barrier growth rate appropriately (i.e., still able maintain a good-quality crystal of the barrier-well heterointerfaces in the MQWs) and reducing the barrier growth time in the high-temperature environment can reduce the thermal budget to mitigate the effect of indium fluctuation. According to the equation of the recombination rate for LEDs (i.e., $\mathrm{R}=\mathrm{An}+\mathrm{Bn}^{2}+\mathrm{Cn}^{3}$ ), the Shockley-Read-Hall (SRH) recombination (An) and the radiative recombination $\left(\mathrm{Bn}^{2}\right)$ are dominant recombination mechanisms at a lower current injection density. Such a result evidences that the LOP and EQE of LED I in Figure 1 are the best at lower injection currents.

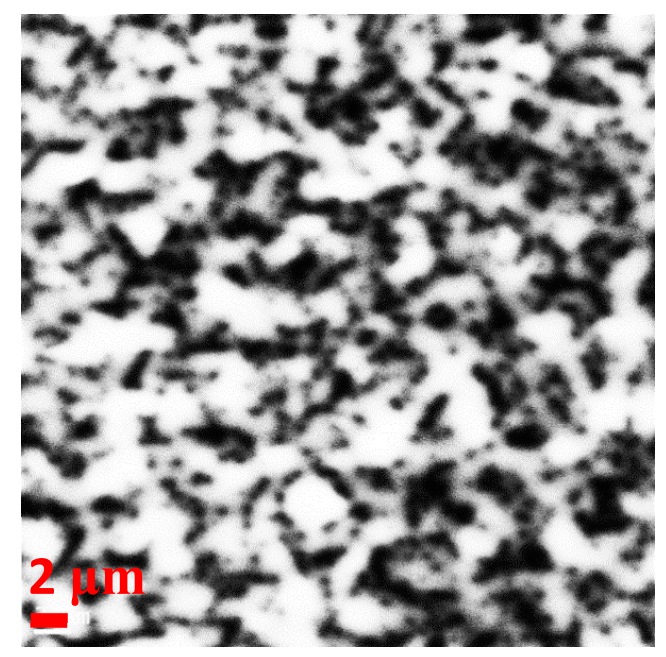

(a)

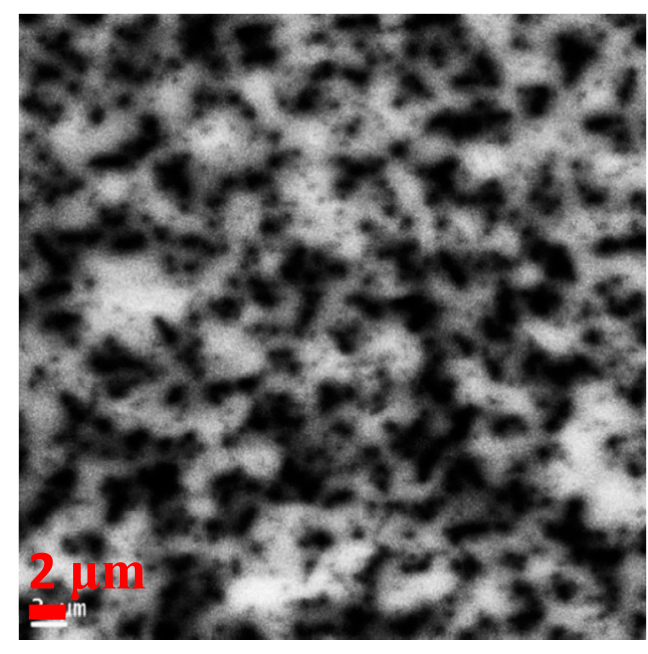

(b)

Figure 4. The CL images in top-view of (a) LED I and (b) LED III.

Figure 5a-c show the temperature-dependent electroluminescence (EL) spectra of LED I, LED II, and LED III, respectively, cooled from $300 \mathrm{~K}$ down to $10 \mathrm{~K}$ at $5 \mathrm{~mA}$ by Helium compressor system and the temperature was controlled by the temperature sensor and heat controller. The similar tendency of temperature-dependent EL intensity of these three fabricated LEDs was observed. As the temperature went down from $300 \mathrm{~K}$, the light intensity of the emission for the LEDs increased. This is attributed to the reduction of the non-radiative recombination rate in the MQW. In other words, the carrier capture rate by the defects will decrease as the temperature decreases [25]. Therefore, the internal quantum efficiency (IQE) of the fabricated LEDs will be increased. Then, the light intensity of the emission began to drop as the temperature kept decreasing due to the reduction of electron and hole concentrations [26]. On the other hand, it was found that the light intensity and FWHM of the emission spectrum for LED III were smaller and wider than those of the other two. In addition to this, it can also be observed that LED III displayed a more evident emission sub-peak than those of the other two around the wavelength of $460 \mathrm{~nm}$. Furthermore, as the ambient temperature decreases, the intensity of the sub-peak has a more significant proportion in the emission spectrum in Figure $5 \mathrm{c}$. This is because when the ambient temperature is lower, the injected carriers into the MQWs are more easily attracted 
and confined in the localized states with lower potential energy and the longer light wavelengths are emitting. It is suggested that the localized states in the InGaN/GaN quantum wells are created by random indium alloy fluctuations or indium-rich clusters [23,24], which could be attributed that the growth temperature of the quantum barriers was $110{ }^{\circ} \mathrm{C}$ higher than that of the quantum wells. As described in the previous paragraph, the higher growth temperature of the quantum barriers can induce indium fluctuation in the MQWs. In addition, the LEDs with quantum barriers grown with a lower growth rate have a larger strain and piezoelectric polarization field between $\mathrm{GaN}$ and InGaN due to the strong lattice mismatch. This is the reason that the lattice constant of the GaN quantum barrier of LED III with a lower growth rate will be closer to the ideal value of GaN bulk than that of LED I, which would result in a larger lattice mismatch between the GaN barrier and InGaN well. Therefore, as the carriers are injected into the MQWs, the localized states with the potential minimum in the QWs created by random indium alloy fluctuations or indium-rich clusters leads to a higher partial carrier density in the QWs, which has a significant effect on the FWHM of the spectrum of emitted light. The Auger recombination procedure in the MQWs is extremely enhanced by the carrier localization mechanism [27]. According to the recombination rate equation of the LEDs (i.e., $\mathrm{R}=\mathrm{An}+\mathrm{Bn}^{2}+\mathrm{Cn}^{3}$ ), the Auger recombination $\left(\mathrm{Cn}^{3}\right)$ is a dominant recombination mechanism at higher current injection density. Thus, the efficiency droop effect induced by Auger recombination will become more serious especially, at higher injection current levels. Therefore, LED III has a more serious carrier localization effect induced by random indium alloy fluctuations. Such a result demonstrates why LED I exhibits the weakest efficiency droop effect in Figure 1b.

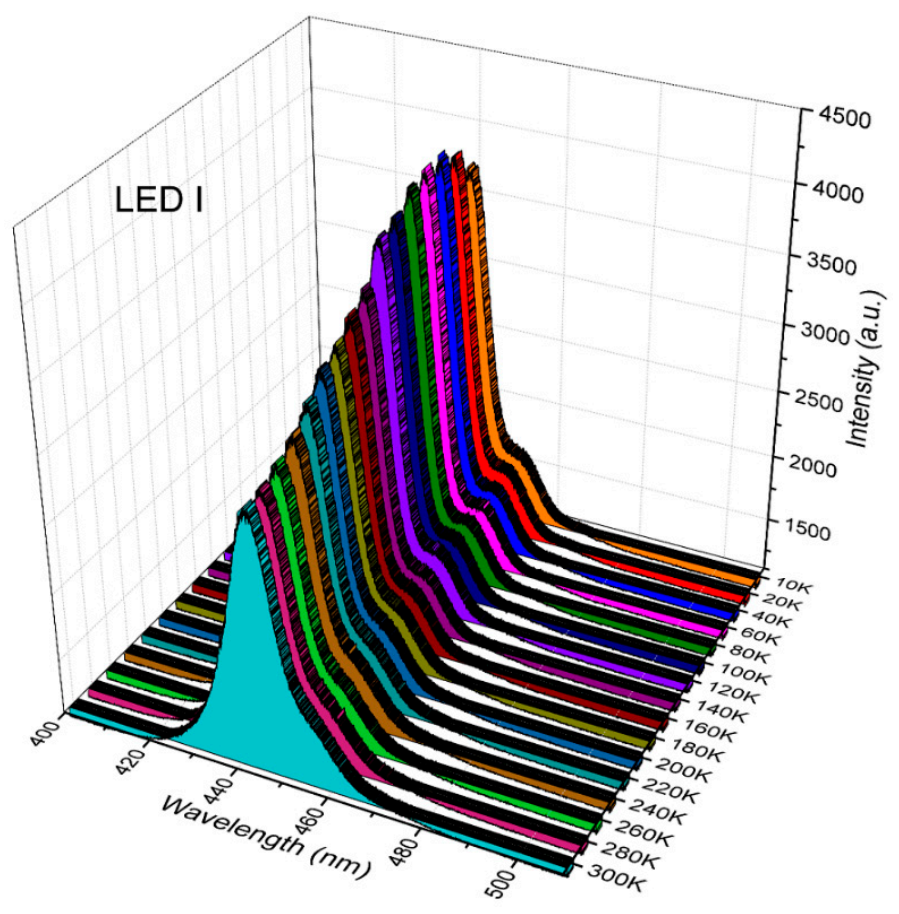

(a)

Figure 5. Cont. 


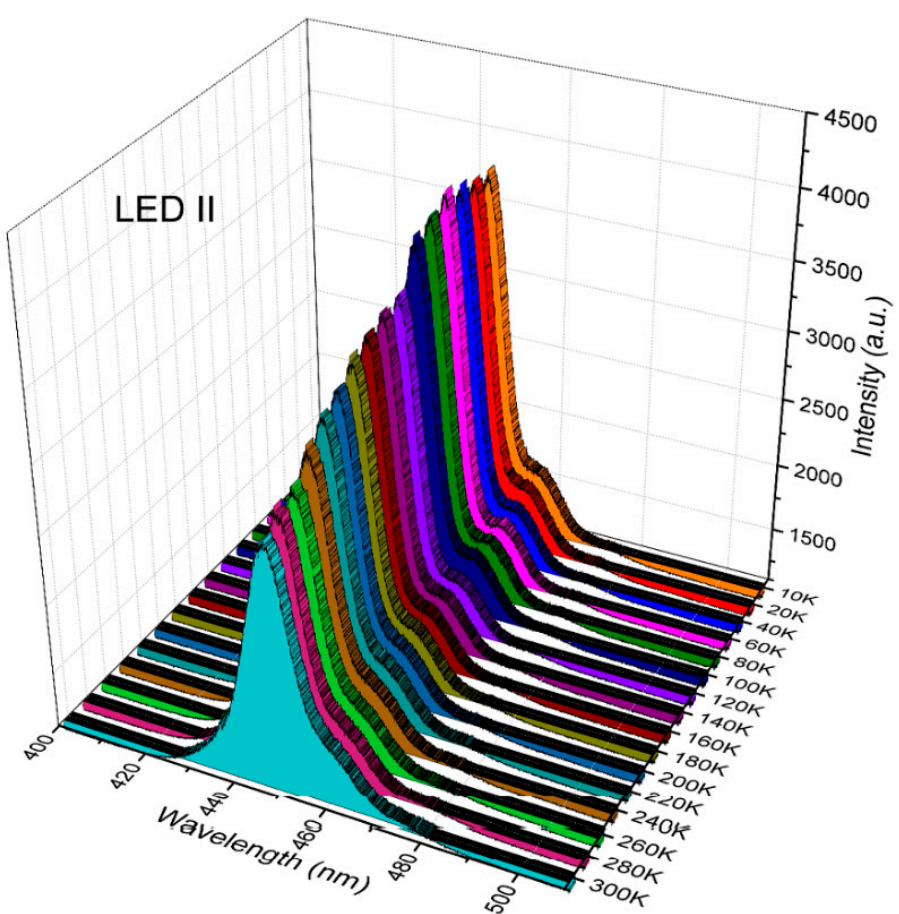

(b)

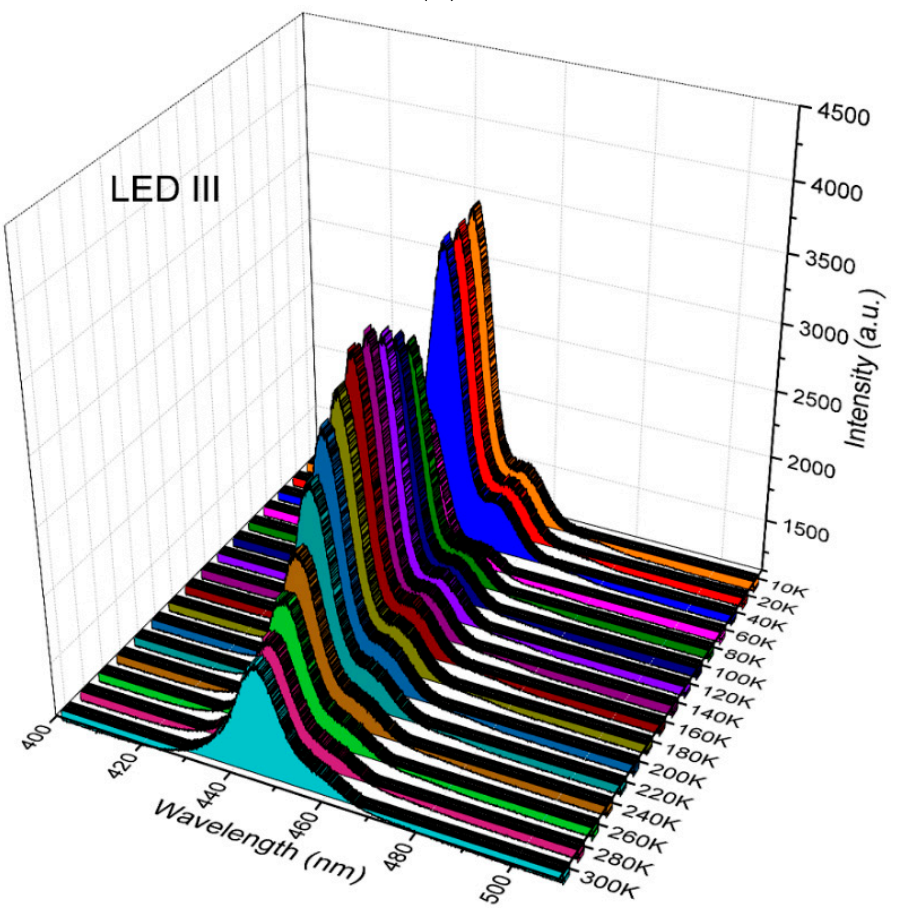

(c)

Figure 5. The EL spectrum of (a) LED I, (b) LED II, and (c) LED III from $10 \mathrm{~K}$ to $300 \mathrm{~K}$ at $5 \mathrm{~mA}$.

\section{Conclusions}

In summary, GaN-based blue InGaN/GaN LEDs with different growth rates of the quantum barriers were demonstrated. It was found that the LOP of the LED with a higher barrier growth rate was $18.4 \%$ higher than the LED with a lower barrier growth rate at $120 \mathrm{~mA}$. The LEDs with quantum barriers grown with a higher growth rate exhibit a lower leakage current and less non-radiative recombination centers in the MQWs from the CL mapping images, which is attributed to a weaker indium fluctuation effect induced by the growth temperature of the quantum barriers $110^{\circ} \mathrm{C}$ higher 
than that of the quantum wells. On the other hand, the localized states in the MQWs created by indium fluctuation lead to a higher local carrier density, which resulted in the Auger recombination in the QWs. Thus, the efficiency droop ratio of the LEDs with a higher barrier growth rate was only $28.6 \%$, which was superior to that with a lower barrier growth rate (39.3\%).

Author Contributions: Conceptualization: C.K.W. and Y.Z.C. Methodology: C.K.W. and Y.Z.C. Validation: C.K.W. and H.J.C. Formal Analysis: C.K.W., Y.Z.C. and H.J.C. Investigation: C.K.W., Y.Z.C. and H.J.C. Resources: C.K.W. and Y.Z.C. Data curation: H.J.C. Writing-original draft preparation, Writing-review and editing: C.K.W. Supervision: C.K.W. and Y.Z.C. Project administration: C.K.W. and Y.Z.C. Funding acquisition: C.K.W.

Funding: This research was funded by Ministry of Science and Technology, Taiwan, grant number: MOST 108-2221-E-218-022.

Acknowledgments: This work was supported by the Ministry of Science and Technology under contract no. MOST 108-2221-E-218-022.

Conflicts of Interest: The authors declare no conflict of interest.

\section{References}

1. Kim, M.H.; Schubert, M.F.; Dai, Q.; Kim, J.K.; Schubert, E.F.; Piprek, J.; Park, Y. Origin of efficiency droop in GaN-based light-emitting diodes. Appl. Phys. Lett. 2007, 91, 183507. [CrossRef]

2. Schubert, M.F.; Xu, J.; Kim, J.K.; Schubert, E.F.; Kim, M.H.; Yoon, S.; Lee, S.M.; Sone, C.; Sakong, T.; Park, Y. Polarization-matched GaInN/AlGaInN multi-quantum-well light emitting diodes with reduced efficiency droop. Appl. Phys. Lett. 2008, 93, 041102. [CrossRef]

3. Zhao, H.P.; Liu, G.Y.; Zhang, J.; Arif, R.A.; Tansu, N. Analysis of internal quantum efficiency and current injection efficiency in III nitride light-emitting diodes. J. Disp. Technol. 2013, 9, 212. [CrossRef]

4. $\quad \mathrm{Ni}, \mathrm{X}$.; Fan, Q.; Shimada, R.; Özgür, Ü.; Morkoç, H. Reduction of efficiency droop in InGaN light emitting diodes by coupled quantum wells. Appl. Phys. Lett. 2008, 93, 171113. [CrossRef]

5. Wang, C.H.; Chang, S.P.; Chang, W.T.; Li, J.C.; Lu, Y.S.; Li, Z.Y.; Yang, H.C.; Kuo, H.C.; Lu, T.C.; Wang, S.C. Efficiency droop alleviation in InGaN/GaN light emitting diodes by graded-thickness multiple quantum wells. Appl. Phys. Lett. 2010, 97, 181101. [CrossRef]

6. Iveland, J.; Martinelli, L.; Peretti, J.; Speck, J.S.; Weisbuch, C. Direct measurement of Auger electrons emitted from a semiconductor light-emitting diode under electrical injection: Identification of the dominant mechanism for efficiency droop. Phys. Rev. Lett. 2013, 110, 177406. [CrossRef]

7. Galler, B.; Lugauer, H.J.; Binder, M.; Hollweck, R.; Folwill, Y.; Nirschl, A.; Gomez-Iglesias, A.; Hahn, B.; Wagner, J.; Sabathil, M. Experimental determination of the dominant type of auger recombination in InGaN quantum wells. Appl. Phys. Express 2013, 6, 112101. [CrossRef]

8. Delaney, K.T.; Rinke, P.; van de Walle, C.G. Auger recombination rates in nitrides from first principles. Appl. Phys. Lett. 2009, 94, 191109. [CrossRef]

9. Yang, Y.; Cao, X.A.; Yan, C. Investigation of the nonthermal mechanism of efficiency rolloff in InGaN light-emitting diodes. IEEE Trans. Electron. Device 2008, 55, 1771. [CrossRef]

10. Harder, J.; Moloney, J.V.; Koch, S.W. Temperature-dependence of the internal efficiency droop in GaN-based diodes. Appl. Phys. Lett. 2011, 99, 181127. [CrossRef]

11. Chow, W.W.; Crawford, M.H.; Tsao, J.Y.; Kneissl, M. Internal efficiency of InGaN light-emitting diodes: Beyond a quasi-equilibrium model. Appl. Phys. Lett. 2010, 97, 121105. [CrossRef]

12. Liu, H.H.; Chen, P.R.; Lee, G.Y.; Chyi, J.I. Efficiency Enhancement of InGaN LEDs with an n-Type $\mathrm{AlGaN} / \mathrm{GaN} / \mathrm{InGaN}$ Current Spreading Layer. IEEE Electron. Device Lett. 2011, 32, 1409. [CrossRef]

13. Zhang, Y.Y.; Yin, Y.A. Performance enhancement of blue light emitting diodes with a special designed AlGaN/GaN superlattice electron-blocking layer. Appl. Phys. Lett. 2011, 99, 221103. [CrossRef]

14. Lin, B.C.; Chen, K.J.; Han, H.V.; Lan, Y.P.; Chiu, C.H.; Lin, C.C.; Shih, M.H.; Lee, P.T.; Kuo, H.C. Advantages of Blue LEDs With Graded-Composition AlGaN/GaN Superlattice EBL. IEEE Photonics Technol. Lett. 2013, 25, 2062. [CrossRef]

15. Park, J.H.; Kim, D.Y.; Hwang, S.; Meyaard, D.; Schubert, E.F.; Han, Y.D.; Choi, J.W.; Cho, J.; Kim, J.K. Enhanced overall efficiency of GaInN-based light-emitting diodes with reduced efficiency droop by Al-composition-graded AlGaN/GaN superlattice electron blocking layer. Appl. Phys. Lett. 2013, 103, 061104. [CrossRef] 
16. Choi, S.; Kim, H.J.; Kim, S.S.; Liu, J.; Kim, J.; Ryou, J.H.; Dupuis, R.D.; Fischer, A.C.; Ponce, F.A. Improvement of peak quantum efficiency and efficiency droop in III-nitride visible light-emitting diodes with an InAIN electron-blocking layer. Appl. Phys. Lett. 2010, 96, 221105. [CrossRef]

17. Keller, S.; Chichibu, S.F.; Minsky, M.S.; Hu, E.; Mishra, U.K.; Den Baars, S.P. Eaect of the growth rate and the barrier doping on the morphology and the properties of InGaN/GaN quantum wells. J. Cryst. Growth 1998, 195, 258. [CrossRef]

18. Schubert, E.F. Light-Emitting Diodes, 2nd ed.; Cambridge Univ. Press: Cambridge, UK, 2006.

19. Lee, S.W.; Oh, D.C.; Goto, H.; Ha, J.S.; Lee, H.J.; Hanada, T.; Cho, M.W.; Yao, T.; Hong, S.K.; Lee, H.Y.; et al. Origin of forward leakage current in GaN-based light-emitting devices. Appl. Phys. Lett. 2006, 89, 132117. [CrossRef]

20. Bandić, Z.Z.; Bridger, P.M.; Piquette, E.C.; McGill, T.C. Minority carrier diffusion length and lifetime in GaN. Appl. Phys. Lett. 1998, 72, 3166. [CrossRef]

21. Ino, N.; Yamamoto, N. Low temperature diffusion length of excitons in gallium nitride measured by cathodoluminescence technique. Appl. Phys. Lett. 2008, 93, 232103. [CrossRef]

22. Brooksby, J.C.; Mei, J.; Ponce, F.A. Correlation of spectral luminescence with threading dislocations in green-light-emitting InGaN quantum wells. Appl. Phys. Lett. 2007, 90, 231901. [CrossRef]

23. Yang, T.J.; Shivaraman, R.; Speck, J.S.; Wu, Y.R. The influence of random indium alloy fluctuations in indium gallium nitride quantum wells on the device behavior. J. Appl. Phys. 2014, 116, 113104. [CrossRef]

24. Islam, A.; Shim, J.I.; Shin, D.S. Optoelectronic Performance Variations in InGaN/GaN Multiple-Quantum-Well Light-Emitting Diodes: Effects of Potential Fluctuation. Materials 2018, 11, 743. [CrossRef] [PubMed]

25. Han, D.P.; Zheng, D.G.; Oh, C.H.; Kim, H.; Shim, J.I.; Shin, D.S.; Kim, K.S. Nonradiative recombination mechanisms in InGaN/GaN-based light-emitting diodes investigated by temperature-dependent measurements. Appl. Phys. Lett. 2014, 104, 151108. [CrossRef]

26. Otsuji, N.; Fujiwara, K.; Sheu, J.K. Electroluminescence efficiency of blue InGaN/GaN quantum-well diodes with and without an n-InGaN electron reservoir layer. J. Appl. Phys. 2006, 100, 113105. [CrossRef]

27. Shahmohammadi, M.; Liu, W.; Rossbach, G.; Lahourcade, L.; Dussaigne, A.; Bougerol, C.; Butté, R.; Grandjean, N.; Deveaud, B.; Jacopin, G. Enhancement of Auger recombination induced by carrier localization in InGaN/GaN quantum wells. Phys. Rev. B 2017, 95, 125314. [CrossRef] 